\title{
Dynamic Activity Dependence of In Vivo Normal Knee Kinematics
}

\author{
Taka-aki Moro-oka, ${ }^{1,2}$ Satoshi Hamai, ${ }^{2}$ Hiromasa Miura, ${ }^{2}$ Takeshi Shimoto, ${ }^{3}$ Hidehiko Higaki, ${ }^{3}$ Benjamin J. Fregly, \\ Yukihide Iwamoto, $^{2}$ Scott A. Banks ${ }^{1,4}$ \\ ${ }^{1}$ Department of Mechanical and Aerospace Engineering, University of Florida, 318 MAE-A, Mail Stop 116250, Gainesville, \\ Florida 32611 \\ ${ }^{2}$ Department of Orthopaedic Surgery, Graduate School of Medical Sciences, Kyushu University, Fukuoka, Japan \\ ${ }^{3}$ Department of Mechanical Engineering, Faculty of Engineering, Kyushu Sangyo University, Fukuoka, Japan \\ ${ }^{4}$ The BioMotion Foundation, Palm Beach, Florida
}

Received 20 November 2006; accepted 25 January 2007

Published online in Wiley InterScience (www.interscience.wiley.com). DOI 10.1002/jor.20488

\begin{abstract}
Dynamic knee kinematics were analyzed for normal knees in three activities, including two different types of maximum knee flexion. Continuous X-ray images of kneel, squat, and stair climb motions were taken using a large flat panel detector. CT-derived bone models were used for model registration-based 3D kinematic measurement. Three-dimensional joint kinematics and contact locations were determined using three methods: bone-fixed coordinate systems, interrogation of CT-based bone model surfaces, and interrogation of MR-based articular cartilage model surfaces. The femur exhibited gradual external rotation throughout the flexion range. Tibiofemoral contact exhibited external rotation, with contact locations translating posterior while maintaining $15^{\circ}$ to $20^{\circ}$ external rotation from $20^{\circ}$ to $80^{\circ}$ of flexion. From $80^{\circ}$ to maximum flexion, contact locations showed a medial pivot pattern. Kinematics based on bone-fixed coordinate systems differed from kinematics based on interrogation of CT and MR surfaces. Knee kinematics varied significantly by activity, especially in deep flexion. No posterior subluxation occurred for either femoral condyle in maximum knee flexion. Normal knees accommodate a range of motions during various activities while maintaining geometric joint congruency. () 2007 Orthopaedic Research Society. Published by Wiley Periodicals, Inc. J Orthop Res 26:428-434, 2008
\end{abstract}

Keywords: tibiofemoral joint; kinematics; dynamic activity; flat panel detector; 3D/2D model registration; tibiofemoral contact

\section{INTRODUCTION}

$3 \mathrm{D}$-to-2D model registration techniques have been used to measure normal in vivo knee kinematics from radiographic images. These studies have provided clinically insightful information and spurred further complementary investigations. Research topics of current interest include exploration of knee kinematics over the full flexion range and development of techniques for generating and reporting data to provide enhanced physiologic insight.

3D kinematics studies have focused on motion from $0^{\circ}$ to $90^{\circ}$ of flexion. Asano et al. ${ }^{1}$ reported a medial pivot motion pattern during static weight bearing from $0^{\circ}$ to $90^{\circ}$. Komistek et al. ${ }^{2}$ reported dynamic normal knee kinematics using shape-

Correspondence to: Scott A. Banks (Telephone: 352-3926109; Fax: 352-392-7303; E-mail: banks@ufl.edu)

๑ 2007 Orthopaedic Research Society. Published by Wiley Periodicals, Inc. matching techniques with computed tomography (CT)-derived bone models. They showed that the lateral condyle experienced significantly more AP translation in activities up to $90^{\circ}$ of flexion with no significant differences among activities. More recently, Li et al. ${ }^{3}$ reported cartilage contact kinematics from $0^{\circ}$ to $90^{\circ}$ using bi-plane fluoroscopy and MRI-derived bone models. No study has analyzed dynamic, weight-bearing tibiofemoral motion from extension to maximum knee flexion.

3D knee kinematic studies also have used a variety of coordinate systems and joint contact computations to describe findings, making it sometimes difficult to compare results of one study to another. For example, varus-valgus motion determined from coordinate systems embedded in the bones can be interpreted to indicate separation of the joint surfaces. However, when the different sagittal slopes of the medial and lateral tibial surfaces are considered, varus-valgus motion can result from the condyles moving AP while remaining in contact with these surfaces. A complete 
mapping of joint surface separations provides a coordinate system-independent measure of articular apposition that avoids misinterpretation. To date, CT-derived bone models (CT model) have been primarily used for model registration-based kinematic measurements. ${ }^{1,2,4}$ Determination of tibiofemoral contact conditions from CT models assumes uniform cartilage thickness. MR-based surface models including cartilage are superior for computing surface interactions, but suffer from less accurate bone definition, which can lead to less precise kinematic measurements. ${ }^{5}$ The optimal approach, therefore, might be to perform model registration using CT-derived bone models with surface interrogations performed using MRderived articular surfaces.

The purpose of this study was to analyze healthy knee kinematics in three activities from extension to maximum knee flexion using high-resolution dynamic flat-panel detector images. A secondary purpose was to consider cartilage thickness in tibiofemoral contact analysis and to compare different methods for reporting articular kinematics.

\section{MATERIALS AND METHODS}

Six healthy male subjects, averaging 29 years (28-31), $171 \mathrm{~cm}(165-177)$, and $69 \mathrm{~kg}(55-80)$ gave informed consent to participate in this study as approved by the institutional review board. Bone models of the femur and tibia/fibula were created from CT (Toshiba, Aquilion, Tochigi, Japan) and MR (Hitachi, Airis II Comfort, Tokyo) scans of one leg. CT scans used a $512 \times 512$ image matrix, a $0.35 \times 0.35$ pixel dim, and a $1-\mathrm{mm}$ thickness spanning approximately $150 \mathrm{~mm}$ above and below the joint line of the knee, and $2 \mathrm{~mm}$ slices through the centers of the hip and ankle. MR scans used a $512 \times 512$ image matrix, a $0.39 \times 0.39$ pixel $\mathrm{dim}$, and a $1-\mathrm{mm}$ thickness spanning more than $80 \mathrm{~mm}$ above and below the joint line of the knee. Scan time of MR ranged from 11 to 15 min. Exterior cortical bone edges for CT and external edges of both cortical bone and cartilage for MRI were segmented using commercial software (SliceOmatic, Tomovision, Montreal, CA), and these point clouds were converted into polygonal surface models (Geomagic Studio, Raindrop Geomagic, Research Triangle Park, NC). Anatomic coordinate systems were embedded in each bone model following conventions of previous studies. ${ }^{5}$ The coordinate systems were first defined for the CT models. The mediolateral (Z) axis of both the femur and tibia/fibula was defined by fitting a cylinder to each posterior condyle of the femur (Fig. 1). The mid point of the cylindrical axis was defined as the coordinate system origin. The proximal/distal $(\mathrm{Y})$ axis for the femur was defined by a line perpendicular to the cylindrical axis in the plane intersecting the femoral head center. The proximal/distal $(\mathrm{Y})$ axis for the shank

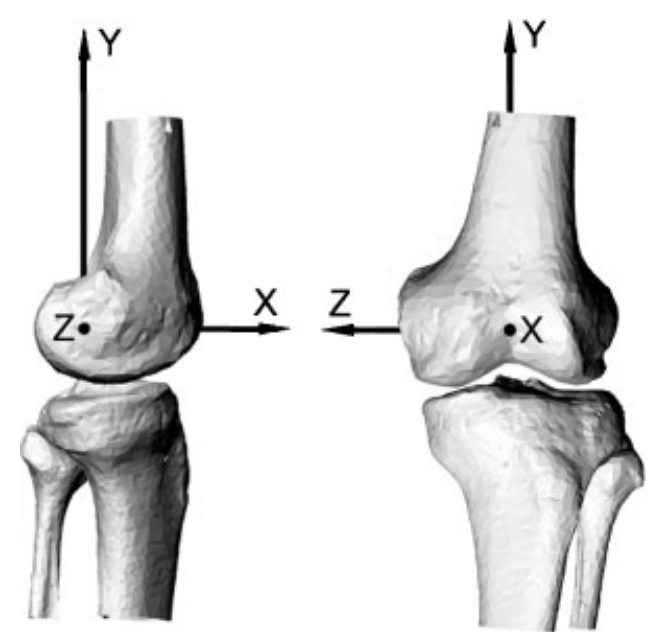

Figure 1. The mediolateral (2) axis of both the femur and tibia/fibula was defined by fitting a cylinder to the posterior condyles of the femur. The mid point of the cylindrical axis was defined as the coordinate system origin for both tibin and femur.

was perpendicular to the cylindrical axis in the plane intersecting the ankle center. The anteroposterior (X) axis was formed from the cross product of the first two. Next, the MR model was registered with the corresponding CT model in its initial reference pose in order to align the embedded coordinate systems in each bone model. Proprietary automated alignment software was used to match 3D bone surfaces (Geomagic Studio). Reported accuracy for this technique is less than 0.1 micrometers in length and 0.1 arcseconds in angle compared to a reference standard value.

Continuous sagittal X-ray images of kneel, squat, and stair-climb activities for each subject were taken using a flat panel detector (Hitachi, Clavis, 3 frames/s, image area $397 \times 298 \mathrm{~mm}, 0.20 \times 0.20 \mathrm{~mm} /$ pixel resolution) (Fig. 2). For the kneel activity, subjects placed their leg on a box, with the foot and ankle hanging freely, and applied their body weight to achieve maximum knee flexion. For the squat activity, the subject stood on a wheeled cart, moved rearward at $>2.5 \mathrm{~cm} / \mathrm{s}$, so their knee position could be maintained in front of the flat-panel detector as they flexed from full extension to full flexion. For the stair activity, subjects ascended a two-step staircase in a reciprocal manner with knee motions recorded on the first step. A total of 293 images were used: 90 images for kneel, 130 for squat, and 73 for stair.

The 3D position and orientation of the tibia/fibula and femur were determined using previously reported shape matching techniques ${ }^{4-6}$ (Fig. 3). A region of the flat-panel X-ray image was extracted and scaled to $512 \times 512$ square pixels for $3 \mathrm{D}$ shape registration. The $\mathrm{CT}$ models were projected onto the X-ray image and manually aligned with the bone projections. An automated matching algorithm using nonlinear least squares optimization, and an image edge-to-model edge distance criteria was used to refine registration of the bone models. RMS errors for this method were $0.53 \mathrm{~mm}$ for in-plane 


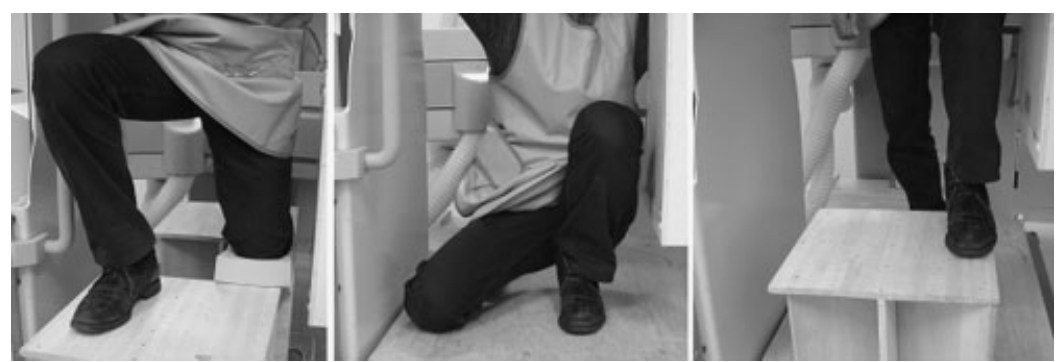

Figure 2. Subjects performed kneeling (left), lunge (middle) and stair (right) activities while their knee motion was observed using a large flat panel x-ray image detector. The images show a left knee being studied. Kneeling was performed from $90^{\circ}$ to maximum comfortable flexion. Squatting was performed from a standing position to maximum comfortable flexion. The stair activity captured the ascent phase from approximately $70^{\circ}$ to $10^{\circ}$ flexion.

translation, $1.6 \mathrm{~mm}$ for out-of-plane translation, and $0.54^{\circ}$ for rotations in a previous study. ${ }^{5}$ Propagation of these uncertainties to the articular surfaces results in a $95 \%$ confidence interval $>2 \mathrm{~mm}$ for declaring separation of the femoral condyles from the tibial surface.

The kinematics of the joint were determined from the $3 \mathrm{D}$ position of each bone model using Cardan angles as described by Tupling and Pierrynowski. ${ }^{7}$ Knee rotations were analyzed as a function of flexion angle. Spline interpolation with $5^{\circ}$ flexion increments was used to create average kinematics for the group. The stair climb proceeded from flexion to extension; the kneel and squat proceeded from extension to flexion. Each subject began and completed each activity at slightly different flexion angles, so averages are reported for flexion angles from three to six knees. One-way ANOVA and post hoc tests (Bonferroni/Dunn) $(p<0.05)$ were used to examine differences among overall results.

The surface of each CT-derived tibial model was divided into medial and lateral compartments and represented as a cloud of points. For every image, a surface separation map was created by computing the minimum distance between each point on the tibial surface and all points on the femoral surface (Fig. 4). Medial and lateral condylar contact locations were computed as the geometric centroid of the region having $<6 \mathrm{~mm}$ separation, which acknowledges uncertainty about cartilage thickness, ${ }^{8,9}$ cartilage deformation, and measurement errors. The choice of separation threshold had a negligible effect on centroid location for thresholds above $3 \mathrm{~mm}$. The angle between the kinematics of the femur with respect to the tibia.

Interactions of cartilage surfaces were determined from the MR-derived models. The bony part of the MR models was registered with the corresponding CT models using automated alignment software (Geomagic Studio). Knee kinematics determined from CT model registration were then used to orient the cartilage surfaces. Contact points were defined in the same manner for the MR models, except a 2-mm maximum separation threshold was used to define the region of contact, contact centroids, and rotational kinematics (Fig. 4).

\section{RESULTS}

Bony kinematics varied among activities. Femoral rotation during squatting was significantly different from kneeling at all flexion angles and differed from stair climbing from $25^{\circ}$ to $35^{\circ}$ flexion (Fig. 5A). However, the femur rotated externally with flexion in all three activities. Sharp external rotation was seen from $0^{\circ}$ to $15^{\circ}$ in squatting,

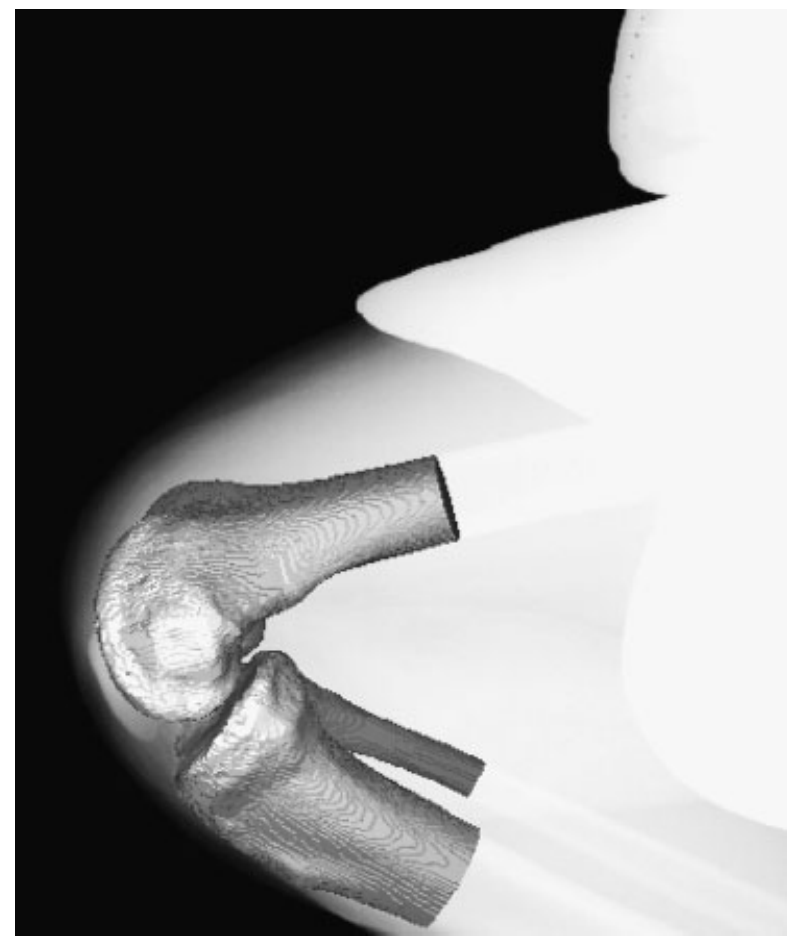

Figure 3. 3D-to-2D CT model-to-flat panel image registration was used to determine the kinematics of healthy knees during three weight bearing activities. 

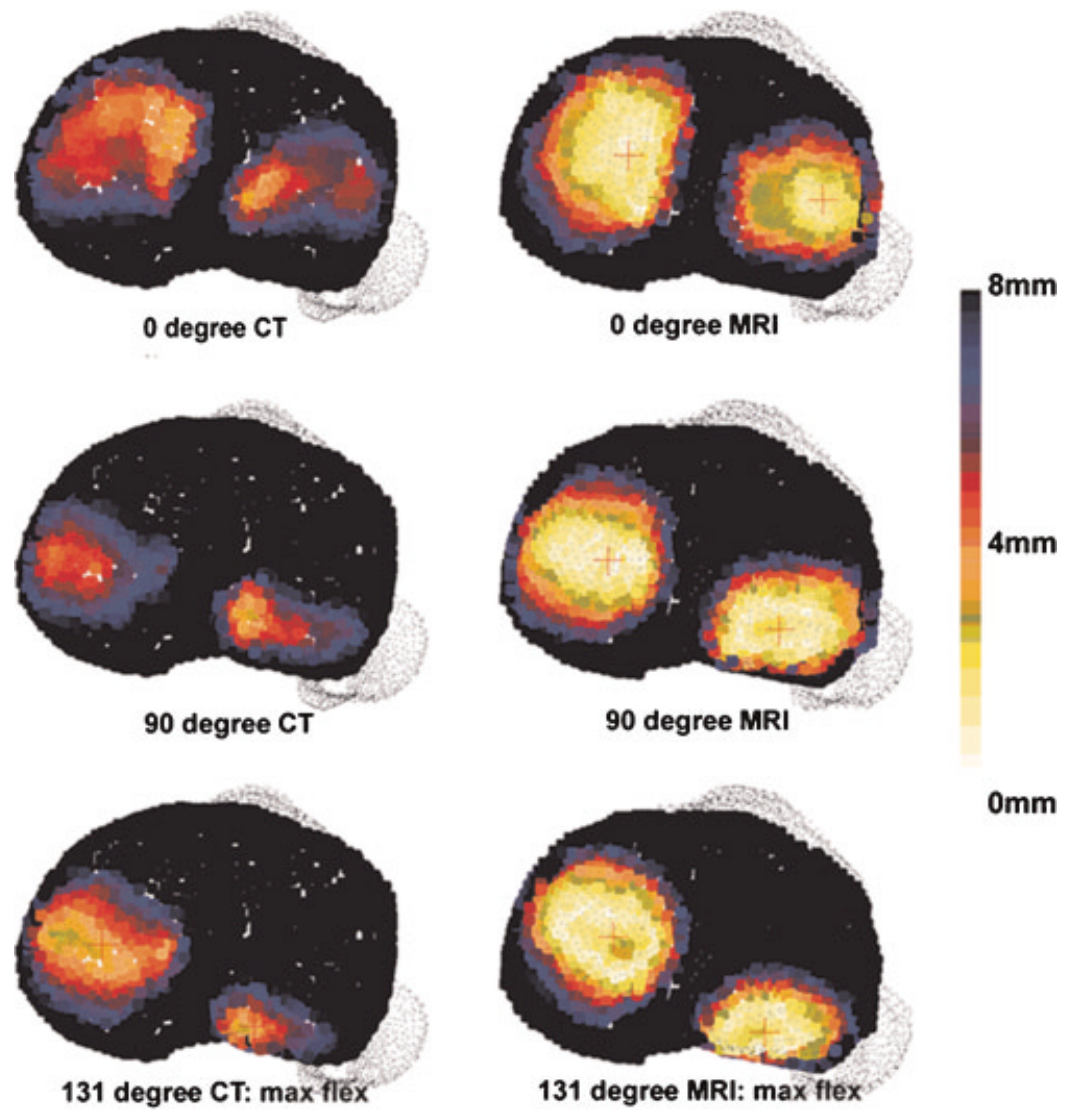

Figure 4. Separation distance mapping for squat at $0^{\circ}, 90^{\circ}$, and maximum flexion $\left(131^{\circ}\right)$ in one subject. Pictures calculated from the MRI model and CT model are shown at the same flexion angle. The centroids of the contact areas are shown as a red cross to indicate the estimated contact location. The picture shows irregular estimated contact regions based on the CT derived models, while the MR derived models of articular cartilage show uniformly rounded contact region estimates

A

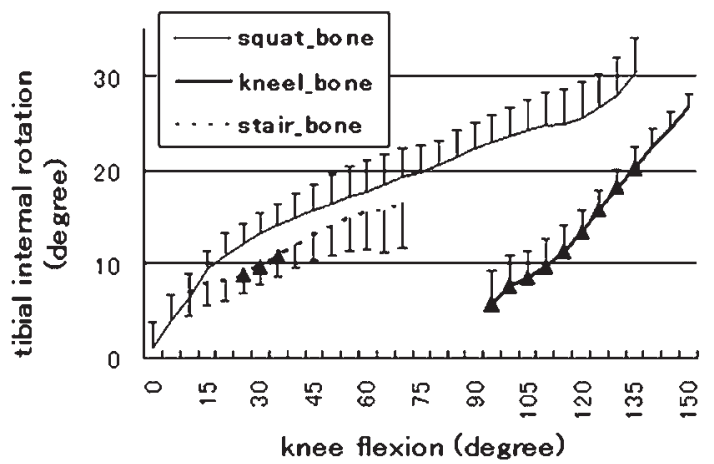

B

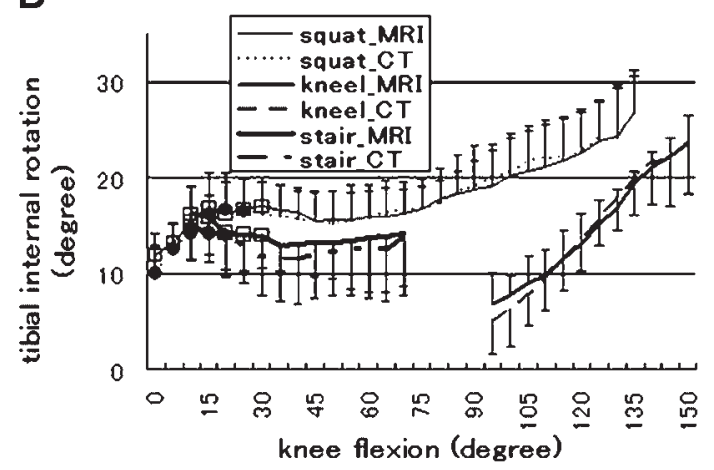

Figure 5. Femoral external rotation increased with knee flexion for stair, squat and kneel activities. A: Femoral external rotations based on bone-embedded coordinate systems. Solid triangles on the stair and kneel represent statistically significant differences from the corresponding rotation during the squat activity $(p<0.05)$. B: Femoral external rotations based on contact locations determined from MR and CT derived cartilage and bone models, respectively. There were no statistically significant differences between rotations measured by these two methods. Solid squares represent significant differences between rotations determined by the MR model based contact locations and the regid body bone motions in graph A $(p<0.05)$. Solid circles represent significant differences between rotations determined by the CT model based contact locations and the rigid body bone motions in graph A $(p<0.05)$. 
similar to the "screw home" movement reported for passive knee motions. ${ }^{10}$

The regions used to estimate contact location were irregularly shaped for the CT models and consistently circular or elliptical for the MR models (Fig. 4). Rotations determined from the $\mathrm{CT}$ and $\mathrm{MR}$ model contact points were not significantly different (Figs. 4 and 5B). There were significant differences between rotations determined from rigid body bone kinematics and contact points for stair and squat activities at flexion angles $<30^{\circ}$ (Fig. 5A and $\mathrm{B})$. There were no statistically significant differences between rotations measured by MR derived model and CT derived model.

AP translation of the condylar contact regions showed three phases over the range of motion (Fig. 6). From extension to $20^{\circ}$ flexion, the contact points rotated externally with posterior translation. From $20^{\circ}$ to $80^{\circ}$, they translated posteriorly with little additional rotation. For flexion $>80^{\circ}$ (squat and kneel), additional external rotation resulted predominantly from posterior lateral translation, essentially pivoting about the medial compartment. There were significant differences in lateral contact positions between squat and kneel from $100^{\circ}-139^{\circ}$ flexion $\left(<0.01\right.$ for $100^{\circ}$ to $135^{\circ}$, and $p=0.015$ at $139^{\circ}$ ). There were no differences in contact positions for the medial condyle.

Condylar separation was not observed at flexion $<150^{\circ}$. In two subjects the minimum medial surface separation exceeded $2 \mathrm{~mm}$ (MR cartilage model) in terminal kneeling at $>150^{\circ}$ flexion.

\section{DISCUSSION}

This study examined dynamic 3D normal knee kinematics in three activities, including two types of maximum knee flexion, using a high-resolution flat panel X-ray detector. Different values of knee axial rotation were observed with different activities, with the greatest femoral external rotation observed during the squat activity. No subluxation of the lateral femoral condyle was observed in maximum knee flexion, and condylar separation from the tibial surface was not commonly observed. Rigid body kinematics measured from bone-embedded coordinate systems produced different joint axial rotations than were measured from the orientation of the medial and lateral condylar contact locations for flexion $<30^{\circ}$.

Knee axial rotations differed according to the method of calculation. Rotations defined from boneembedded coordinate systems showed gradual femoral external rotation across the entire flexion arc (Fig. 5A). Rotations measured from condylar
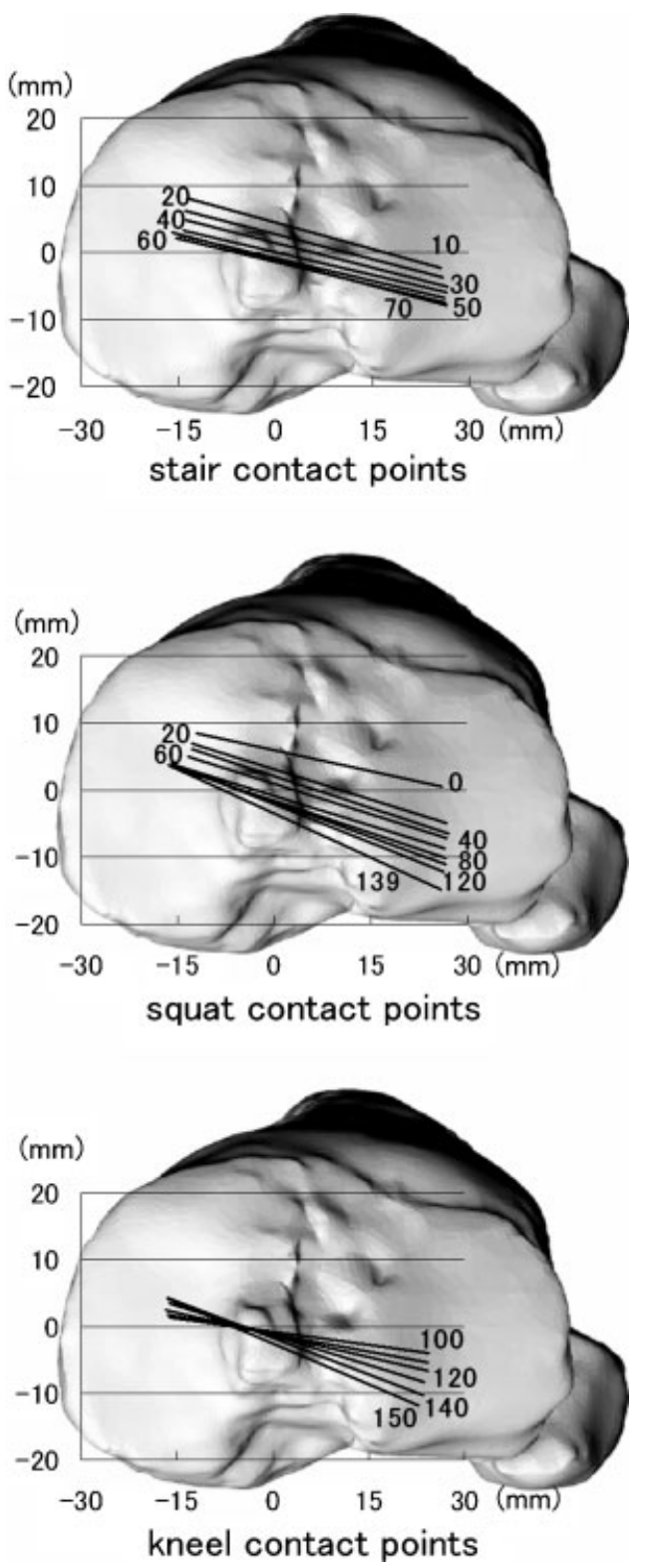

Figure 6. Average contact locations superimposed on an MR model of the tibial surface. For all activities, the contact points in the lateral compartment moved from anterior to posterior with knee flexion. Contact locations are shown for $10^{\circ}$ flexion increments for stair and kneel activities, $20^{\circ}$ flexion increments for the squat activity. There were no significant differences between contact locations for the squat and stair activites.

contact locations exhibited three phases: sharp external rotation from $0^{\circ}$ to $15^{\circ}$ flexion, relatively constant rotation from $20^{\circ}$ to $80^{\circ}$ flexion, and increasing rotation from $80^{\circ}$ to $150^{\circ}$ flexion (Fig. 5B). Significant differences in early flexion $\left(0^{\circ}\right.$ to $\left.30^{\circ}\right)$ are due to femoral geometry. Iwaki et al. ${ }^{11}$ showed that the sagittal profile of the medial femoral condyle is composed of a larger distal and smaller posterior circular arc, while the lateral condyle is well described by a single circular facet. 
From extension to mid flexion, the medial tibial contact area had a more ovoid shape and moved posteriorly (Figs. 5 and 6). This relative internal rotation of the contact locations was offset by relative external femoral rotation, resulting in less rotation measured at the contact locations than was measured from the bone-embedded coordinate systems over this range. From $20^{\circ}$ to $80^{\circ}$ flexion the contact locations moved posterior, inclined $15^{\circ}$ to $20^{\circ}$ with respect to the tibia, maintaining the femur approximately in line with the foot as previously reported..$^{11}$ However, unlike previous reports, contact-derived rotation continued from $80^{\circ}$ to full flexion in squat and kneel. From $80^{\circ}$ to maximum flexion, the contact-derived and rigid body bone rotations showed similar curves, which is reasonable considering the circular geometry of the posterior femoral condyles. ${ }^{11}$

Banks et al. ${ }^{12}$ showed kinematics varied with implant design and activity in total knee arthroplasty patients. In this study, kinematics varied with activity in healthy knees, particularly with squat and kneel. The greatest total rotation and the most posterior lateral contact was observed with squatting, but the maximum flexion angle and the rate of tibial rotation from $100^{\circ}$ to maximum flexion was greater with kneeling. Medial contact was in the same area for both activities. The lateral femoral condyle translated to the posterior edge of the tibial surface during squat, although there was no subluxation. Subjects were encouraged to achieve comfortable maximum flexion, thus results may have differed if the squat was forced to greater knee flexion.

Comparison of stair climb and squat activities revealed small kinematic differences in the middle range of flexion, which were not statistically significant. The femur showed greater external rotation for all flexion angles during squatting. Results from cadaver studies, ${ }^{11}$ MRIs of living knees, ${ }^{13}$ and living knees with $\mathrm{RSA}^{14}$ have shown that a posture with tibial external rotation can suppress tibial internal rotation over the flexion arc. The wider stance width used during the squat activity probably biased the knee toward greater femoral external rotation in the mid range of flexion. Standard deviations for the squat and stair activities were $20 \%$ greater than for the kneel activity, suggesting kneeling is more geometrically constrained or stereotypic.

Surface distance calculations revealed only two cases showing evidence of condylar separation, both occurring at terminal flexion in kneeling beyond $150^{\circ}$. In the static MRI study of maximally flexed knees, Nakagawa et al. ${ }^{15}$ reported the medial condyle remained positioned over the tibial articular surface but lifted away from it, mechanistically similar to the present finding. Two differences may account for infrequent observation of condylar separation in this study. First, a conservative threshold (95\% confidence interval) was used for declaring separation, acknowledging uncertainty accrued from measurement uncertainty, surface modeling errors, and possible cartilage deformation. Second, Nakagawa et al. ${ }^{15}$ studied a different activity. In this study, subjects knelt to maximum passive flexion with partial body weight and an upright trunk posture (hip angle approximately $90^{\circ}$ ). The subjects reported in Nakagawa et al. ${ }^{15}$ were supine, with the hip extended in the MRI scanner, similar to an athlete's quadriceps stretching posture. Hip extension might have caused greater quadriceps tension and resulted in a more exaggerated joint posture.

Nakagawa et al. ${ }^{15}$ also concluded that the lateral femoral condyle subluxed posteriorly from the tibia at full passive flexion, a phenomenon not seen in the present study. Nakagawa et al. ${ }^{15}$ tracked a cylindrical axis embedded in the posterior condyles and determined that subluxation occurred when the lateral axis moved posterior to the tibial surface at $162^{\circ}$ flexion. However, subluxation is an articular phenomenon where the surfaces of a joint no longer face each other exactly but remain partially aligned. MR and cryosection pictures in the report of Nakagawa et al. ${ }^{15}$ show the lateral femoral condyle has adequate contact with the edge of the tibial articular surface even when the cylindrical axis has exceeded the tibial periphery. These pictures are consistent with the observations of this study.

Findings of articular separation and subluxation in healthy joints sometimes can result from methodological issues and sometimes from articular reality. In the latter case, it these phenomena might be better appreciated as the boundaries of the passive characteristics of the normal healthy joint than negative attributes. For example, the healthy knee has been observed to have articular gaps in $90^{\circ}$ of flexion in the medial compartment and in the lateral compartment depending on the posture. ${ }^{16}$ The knee joint has long been known to possess an envelope of possible motions that only now are being explored by advanced measurement techniques.

The combination of dynamic imaging with flatpanel detectors and CT/MR bone models provide some novel measurement opportunities. However, refinements and enhancements to the method will expand the range of possible studies. First, 
the flat-panel detector used for this study provided high spatial resolution but only three images/s. Solid-state detectors now commercially available with 30 images/s capabilities will permit study of highly dynamic activities. Second, single-plane imaging provides tremendous experimental flexibility and relatively large viewing volumes, but suffers from the main limitation of monocular vision-poor sensitivity for motions perpendicular to the image plane, which was approximately $2 \mathrm{~mm}$ in our preliminary study using a lower resolution image source. ${ }^{5}$ Bi-plane imaging techniques can provide measurements with enhanced precision and more uniform uncertainties when required, but restrict the viewing volume, increase X-ray exposure and at least double the cost. Third, geometric and coordinate alignment errors in the CT and MR models affect the measurement accuracy, and it is well known that MR images/ models suffer from geometric distortion. ${ }^{5}$ For this reason, CT-derived models were used for 3D-to-2D model-to-image registration, only using the MRderived models for articular surface analysis once the $3 \mathrm{D}$ pose of the bones was determined. In addition, the MR scans were performed nonweight bearing, so the compliant cartilage surfaces presumably were unloaded. Additional measurement uncertainty results when interrogating these cartilage surfaces based on weight-bearing conditions, ${ }^{17}$ and the contact locations must be defined conservatively. Fourth, the current approach to analyzing joint contact ignores the menisci, which are invisible on X-ray but obviously affect joint contact and load distribution. There is not currently an X-ray-based technique that will overcome this limitation, but dynamic MR studies can provide this capability. ${ }^{18}$ Finally, simple experimental issues limited the range of the current observations. Subjects were instructed to perform the three activities in a natural and comfortable manner, and some subjects extended and/or flexed their knees more than others. The data reported here are limited to the common flexion ranges for the subjects. In summary, the study provides observations of healthy knee kinematics in three different activities over the flexion range from extension to $150^{\circ}$ using dynamic imaging with CT/MR-derived models. Future experimental and technical improvements might further expand the range and quality of measurements that can be obtained.

\section{REFERENCES}

1. Asano T, Akagi M, Tanaka K, et al. 2001. In vivo three-dimensional knee kinematics using a biplanar image-matching technique. Clin Orthop Relat Res 388:157-166.

2. Komistek RD, Dennis DA, Mahfouz M. 2003. In vivo fluoroscopic analysis of the normal human knee. Clin Orthop Relat Res 410:69-81.

3. Li G, DeFrate LE, Park SE, et al. 2005. In vivo articular cartilage contact kinematics of the knee: an investigation using dual-orthogonal fluoroscopy and magnetic resonance image based computer models. Am J Sports Med 33:102107.

4. Fregly BJ, Rahman HA, Banks SA. 2005. Theoretical accuracy of model-based shape matching for measuring natural knee kinematics with single-plane fluoroscopy. J Biomech Eng 127:692-699.

5. Moro-oka T, Hamai S, Miura H, et al. 2007. Can MR derived bone models be used for accurate motion measurement with single-plane 3D shape registration? J Orthop Res 7:867-872.

6. Banks SA, Hodge WA. 1996. Accurate measurement of three-dimensional knee replacement kinematics using single-plane fluoroscopy. IEEE Trans Biomed Eng 43:638-649.

7. Tupling SJ, Pierrynowski MR. 1987. Use of cardan angles to locate rigid bodies in three-dimensional space. Med Biol Eng Comput 25:527-532.

8. Adam C, Eckstein F, Milz S, et al. 1998. The distribution of cartilage thickness in the knee-joints of old-aged individuals-measurement by A-mode ultrasound. Clin Biomech (Bristol, Avon) 13:1-10.

9. Koo S, Gold GE, Andriacchi TP. 2005. Considerations in measuring cartilage thickness using MRI: factors influencing reproducibility and accuracy. Osteoarthritis Cartilage 13:782-789.

10. Moglo KE, Shirazi-Adl A. 2005. Cruciate coupling and screw-home mechanism in passive knee joint during extension-flexion. J Biomech 38:1075-1083.

11. Iwaki H, Pinskerova V, Freeman MA. 2000. Tibiofemoral movement 1: the shapes and relative movements of the femur and tibia in the unloaded cadaver knee. J Bone Joint Surg Br 82:1189-1195.

12. Banks SA, Hodge WA. 2004. 2003 Hap Paul Award Paper of the International Society for Technology in Arthroplasty. Design and activity dependence of kinematics in fixed and mobile-bearing knee arthroplasties. J Arthroplasty 19:809-816.

13. Hill PF, Vedi V, Williams A, Iwaki H, et al. 2000. Tibiofemoral movement 2: the loaded and unloaded living knee studied by MRI. J Bone Joint Surg Br 82:11961198.

14. Karrholm J, Brandsson S, Freeman MA. 2000. Tibiofemoral movement 4: changes of axial tibial rotation caused by forced rotation at the weight-bearing knee studied by RSA. J Bone Joint Surg Br 82:1201-1203.

15. Nakagawa S, Kadoya Y, Todo S, et al. 2000. Tibiofemoral movement 3: full flexion in the living knee studied by MRI. J Bone Joint Surg Br 82:1199-1200.

16. Tokuhara Y, Kadoya Y, Nakagawa S, et al. 2004. The flexion gap in normal knees. An MRI study. J Bone Joint Surg Br 86:1133-1136.

17. Forster H, Fisher J. 1996. The influence of loading time and lubricant on the friction of articular cartilage. Proc Inst Mech Eng [H] 210:109-119.

18. Sheehan FT, Zajac FE, Drace JE. 1999. In vivo tracking of the human patella using cine phase contrast magnetic resonance imaging. J Biomech Eng 121:650-656. 